\title{
Allo stato, possiamo dare via libera!
}

Vittorio Coletti

PUBBLICATO: 18 FEBBRAIO 2020

\section{Quesito:}

Ci ha scritto un lettore, da Taranto, che sente spesso giornalisti e avvocati utilizzare frasi del tipo: "Allo stato, non risultano prove ..." e ci chiede: "È corretta questa forma? Non è priva di senso? Non si dovrebbe dire allo stato attuale?".

\section{Allo stato, possiamo dare via libera!}

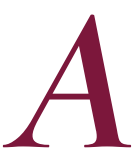

llo stato è una locuzione avverbiale in cui stato significa 'condizione', 'modo di essere', 'situazione', in genere specificati da aggettivi (attuale, presente) o complementi (delle cose, delle conoscenze), ma oggi ormai anche sottintesi. Ha un valore temporale e limitativo insieme, traducibile con 'attualmente', 'per quello che se ne sa', 'per quanto risulta', ed esprime sincronia con ciò di cui si parla e limitazione temporale della sua validità. Il suo uso, come ha notato anche il nostro lettore, è segnalato soprattutto in ambito amministrativo e giuridico: atti parlamentari: "premesso che: nel territorio di Sessa Aurunca è stato realizzato un nuovo ospedale allo stato non ancora entrato in funzione..." (Camera dei Deputati, Discussioni 1989), comunicati di Enti: "Allo stato non risultano essere notificati né all'Ente né alla Società di Gestione, provvedimenti di sequestro del depuratore" (Comune di Borgia, 29 novembre 2019), "Allo stato, non risultano coinvolti automezzi e persone" (Autostrada dei Fiori, 24 novembre 2019), sentenze: "rilevato altresi che allo stato non risultano elementi per pronunciare sentenza di proscioglimento" (Iaselli, Codice di procedura penale illustrato, 2014), ma si può trovare anche in diverse situazioni ad alta formalità comunicativa (ad esempio, diagnosi, referti medici: "Allo stato, non apprezzabili aree focali di restrizione della diffusività a carico del tessuto encefalico", da Pazienti.it). Il Vocabolario Treccani online e lo Zingarelli zorg la classificano come burocr(atica).

È ammissibile, è sensata?, si chiede il nostro lettore. Sul web non mancano riprovazioni. Si ricusa la forma ellittica, chiedendo che lo stato sia, per cosi dire, esplicitato, completato, come nella forma analoga allo stato dell'arte, cioè delle conoscenze, un calco formale (ma non semantico) di una locuzione inglese. In realtà, è abbastanza frequente lo sviluppo ellittico di unità polirematiche (insiemi unitari di parole distinte). Ad esempio, se diciamo che "è in corso un controllo della finanza" è perché la polirematica "guardia di finanza" si è risolta con l'ellissi della sua testa. Come altre locuzioni, dunque, anche la nostra è ellittica, cioè sottintende qualcosa con cui si trova spesso. Si diceva, un aggettivo o un complemento (che serve a ricordarci anche come all'origine si tratti di una locuzione preposizionale): allo stato delle cose, dei fatti, delle conoscenze, degli atti ecc., in espressioni a loro volta ellittiche (allo stato delle cose $=$ allo stato in cui si trovano le cose). L'ellissi è presente in locuzioni avverbiali concorrenti, come al momento (= al momento attuale) o al(lo stato) presente. A ben vedere ci sono altre locuzioni avverbiali temporali che sottintendono parole o addirittura frasi, come allepoca, al tempo (sottinteso: di cui si parla). Se vogliamo, persino il comunissimo allora discende (e contiene in sé i resti) di una locuzione latina, ad illam horam 'a quell'ora', il cui senso è completato dal sottinteso (di cui si tratta, si sta parlando qui ecc.), cioè da elementi che ne spiegano il successivo sviluppo come connettivo testuale. Lo stesso si può dire della locuzione antiquata al postutto 'dopo tutto' (sottinteso: quello che è stato detto). Ma in questi ultimi casi è sottintesa un'intera frase. Nel nostro allo stato, 
come in genere nelle polirematiche, è invece sottinteso un elemento nominale ricorrente. Ora, l'ellissi viene dopo una lunga esplicitazione dei componenti poi sottintesi ed è quindi in genere più recente. Si nota anche nel nostro caso. Pur ricorrendo a Google Libri non sono riuscito a raccogliere attestazioni di allo stato precedenti alla fine degli anni Settanta ("La richiesta deve essere rigettata, perché allo stato non risultano elementi di tale gravità da giustificare la sottrazione del processo al giudice naturale", da "Il Foro italiano. Raccolta generale di giurisprudenza", 1979), anche se il loro numero cresce rapidamente in seguito. La forma ellittica non si trova ovviamente nei corpora antichi né in alcun testo del corpus DiaCORIS. Anche nel corpus PTLLIN, stato è sempre specificato da aggettivi o complementi. In Google la frase "allo stato non risultano" nel senso qui inteso (cioè 'attualmente non risultano') ha varie migliaia di occorrenze e tutte assai recenti, la maggior parte degli anni Duemila.

Insomma, è un nuovo, fortunato sviluppo lessicale, proprio della lingua amministrativa e giuridica, che ricorre volentieri ad accorciamenti, tagli, ellissi, acronimi. Poiché non è equivoca, essendo formalmente corretta, anche la forma allo stato è accettabile. Ma laddove si potrebbe equivocare stato col suo significato di ente giuridico e quindi, ad esempio, intendere la frase "allo stato non risulta" come "allo Stato non risulta", specificare con un aggettivo o complemento di che stato si tratti (attuale, delle cose, delle ricerche ecc.) costa poco e non è male. Non di rado, l'espressione allo stato è seguita nello scritto da una virgola, a segnalare la sua natura avverbiale ed evitare così ogni equivoco.

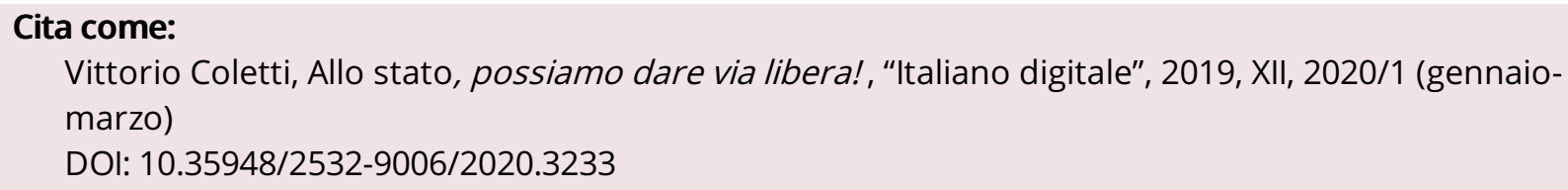

